\title{
Intracytoplasmic sperm injection outcomes after anti-oxidant treatment in repeated implantation failure
}

\author{
Semih Tangal ${ }^{1} \odot$, Gamze Sinem Çağlar $^{2} \odot$, Emre Göksan Papuçcu²$^{2}$, Müge Keskin ${ }^{2} \odot$, Ahmet Hakan \\ Haliloğlu1 ${ }^{1}$
}

${ }^{1}$ Department of Urology, Ufuk University School of Medicine, Ankara, Turkey, Turkey

${ }^{2}$ Department of Obtetrics and Gynecology, Ufuk University School of Medicine, Ankara, Turkey

DOI: $10.18621 /$ eurj.429257

\begin{abstract}
Objectives: Aim of this study was to explore the effects of three months oral anti-oxidant treatment in normozoospermic men with high DNA fragmentation index (DFI) and recurrent intracytoplasmic sperm injection (ICSI) failures.

Methods: This is a retrospective analyses of normozoospermic patients with high DFI levels ( $>30 \%)$ with at least two unsuccessful ICSI attempts. Among the participants, the study group included men who had oral antioxidant treatment. The men who did not receive any treatment during the study period were taken as controls. The outcomes of new ICSI cycles were compared.

Results: During the study period 24 men have used three months of oral anti-oxidant treatment before a new ICSI cycle whereas the remaining 46 had ICSI-only without any other intervention. Duration of stimulation, the number of metaphase II oocytes, fertilization rate and implantation rates were similar between two groups. Although not statistically significant, cases in anti-oxidant group have slightly better pregnancy rates compared to ICSI-only group $(37.5 \%$ vs $19.6 \%, p>0.05)$. Only one case in each group had abortion.

Conclusions: This study reveals that there is no statistically significant difference in the ICSI outcomes of the patients with high DFI levels, following unsuccessful ICSI attempts after oral anti-oxidant treatment. The possible benefit derived from the anti-oxidant therapy needs to be clarified with clinical studies with greater study populations.
\end{abstract}

Keywords: Oxidative stress, DNA fragmentation, intracytoplasmic sperm injection, spermatozoa

Received: May 31, 2018; Accepted: August 10, 2018; Published Online: May 10, 2019

\begin{abstract}
A mong reproductive age couples, approximately $15 \%$ suffer from infertility [1]. A relatively high number of women fail to have a pregnancy despite the apperent absence of a male or female factor of infertility. Normal semen analysis in these couples mandates some other analyses which address the integrity of sperm DNA. Recent studies have documanted an
\end{abstract}

association between high levels of sperm DNA fragmentation and impaired fertilization/embryo development, lower implantation rates and higher miscarriages [2]. In animal studies, sperm DNA damage vas found to be related with birth defects in the offsprings due to impaired DNA integrity [3]. DNA damage in spermatozoa may be induced by different

Address for correspondence: Semih Tangal,MD., Asistant Professor, Ufuk University School of Medicine, , Department of Urology, Ankara, Turkey E-mail: semihtangal@gmail.com 
mechanisms that include; apoptosis, abnormal chromatin packaging and post-testicular causes of sperm DNA fragmentation ensued from reactive oxygen species (ROS) during transit through the mail reproductive tract, DNA fragmentation caused by endogenous endonucleases, radiotherapy and chemotherapy or enviromental causes (smoking, pollution) [4].

Oxidative stress to spermatozoa is very common, affecting between $30 \%$ and $80 \%$ of infertile men [5]. Seminal plasma the primary source of ROS is spermatozoa and polymorphonuclear leukocytes. In fact production of ROS is an outgrowth of physiological process during capacitation and acrosome reaction. Yet, high levels of ROS in seminal plasma is associated with poor semen quality. In one study it has been demonstrated that ROS causes almost 4-fold higher levels of DNA fragmentation and can be treated with anti-oxidants [6].

Antioxidant treatment has been widely used and accepted treatment for men with high sperm DNA fragmentation [7]. Several studies have suggested the use of anti-oxidant therapy in the treatment of male infertility to improve semen quality, especially in DNA damage and sperm motility [8]. Aim of this study was to explore the effects of three months oral anti-oxidant treatment in men with high levels of sperm DNA fragmentation and normozoospermia and history of recurrent intracytoplasmic sperm injection (ICSI) failures.

\section{METHODS}

This is a retrospective analyses of patients who admitted to private in vitro fertilization (IVF) center between January and December 2016. This study included normozoospermic patients (according to WHO 2010 criteria) with high DFI levels (> 30\%) detected by TUNEL assay. All patients had experienced at least two unsuccessful ICSI attempts with ejaculated spermatozoa. Among the participants, the study group included men who had oral antioxidant treatment. The men who did not receive any treatment during the study period were taken as controls. Heavy smokers ( $>20$ cigarettes/day) and patients with; abnormal medical history, abnormalities physical examination, impaired endocrine profile, genital infections, leukocytospermia, cancer or cancer therapies, varicocele, cryptorcidism and genetic disorders were excluded. Couples with female partners fulfilling the Bologna criteria, preimplantation genetic screening and cryo cycles were also excluded.

All participants underwent ICSI using antagonist protocol. On day 2 of menstruel cycle, gonadotropins were started at doses ranging from 150 to 225 IU (Menopur, Ferring). On day 6 Gonadotropin-releasing hormone $(\mathrm{GnRH})$ antagonist was added $(0.25 \mathrm{mg} /$ day, Cetrotide, Serono). The gonadotropin doses are adjusted according to patients response. When at least three follicules are $>17 \mathrm{~mm}$ in diameter ovulation is triggered with human chorionic gonadotropin (hCG) (Ovitrelle, Serono). Oocyte pick up is performed under sedation. The number of metaphase II (MII) oocytes, fertilization, embryo quality, and the cycle outcomes (implantation, clinical pregnancy, early pregnancy loss) of all couples were recorded. Patients in the study group underwent ICSI after receiving 3 months of anti-oxidant treatment (Proxeed Plus, İstanbul, Eczacıbaş1), while ICSI was the sole treatment for controls. Pregnancy was recorded after betahCG test at day 12 after embryo transfer.

This retrospective study was conducted in accordance with the ethical principles defined in the Helsinki Declaration.

\section{Statistical Analysis}

Statistical analyses performed by IBM SPSS for Windows Version 22.0 statistical package. Continuous variables presented as mean \pm standard deviation or median (minimum-maximum). Categorical variables summarized as frequencies and percentages. Normality of the continuous variables was evaluated by Kolmogorov Smirnow test. Differences between the two groups according to continuous variables were determined by independent samples t-test or Mann Whitney U test as appropriate. Categorical variables were compared by Chi-square or Fisher's exact test. Univariate associations between variables were estimated by Pearson's coefficient correlation. A p value less than 0.05 was considered significant.

\section{RESULTS}

Analysis of the recorded revealed that there are 70 couples fulfilling the inclusion criteria. Among these cases 24 men have used three months of anti-oxidant 
Table 1. Demographic characteristics of groups

\begin{tabular}{lccc}
\hline & $\begin{array}{c}\text { OA } \\
(\mathbf{n = 2 4 )}\end{array}$ & $\begin{array}{c}\text { ICSI-only } \\
(\mathbf{n}=\mathbf{4 6})\end{array}$ & $\boldsymbol{p}$ value \\
\hline Age of females (years) & $33.25 \pm 5.18$ & $34.1 \pm 3.68$ & 0.423 \\
BMI $\left(\mathrm{kg} / \mathrm{m}^{2}\right)$ & $24.33 \pm 3.17$ & $25.76 \pm 2.36$ & 0.170 \\
Previous failed attempts (n) & $3.17 \pm 1.55$ & $3.86 \pm 1.42$ & $<\mathbf{0 . 0 5}$ \\
Infertility duration(years) & $7.21 \pm 2.78$ & $8.2 \pm 2.60$ & 0.144 \\
AMH (ng/ml) & $2.20 \pm 1.39$ & $2.12 \pm 1.16$ & 0.810 \\
DFI (\%) & $43.33 \pm 5.87$ & $41.8 \pm 6.48$ & 0.337 \\
\hline
\end{tabular}

Data are presented as mean \pm standard deviation. $\mathrm{OA}=$ oral antioxidant, ICSI $=$ intracytoplasmic sperm injection, $\mathrm{BMI}=$ Body mass index, $\mathrm{AMH}=$ Anti-Mullerian Hormone, $\mathrm{DFI}=\mathrm{DNA}$ fragmentation index

treatment before a new ICSI cycle whereas the remaining 46 had ICSI-only. The study and control groups had comparable demographic characteristics by means of female partner's age, body mass index (BMI), duration of infertility, anti-Mullerian hormone (AMH) levels and DNA fragmentation index (DFI); suggesting that the confounding factors supposed to influence ICSI outcomes were almost matched (Table 1). The only statistically significant differences between the treatment and control groups were the number of previous failed attempts.

The cycle characteristics and outcomes are given in Table 2. Duration of stimulation, the number of MII oocytes, fertilization rate and implantation rates were similar between two groups. Although not statistically significant, cases in anti-oxidant group have slightly better pregnancy rates compared to ICSI-only group $(37.5 \%$ vs $19.6 \%, p>0.05$, Table 2$)$. Only one case in each group had abortion.

\section{DISCUSSION}

Although there have been previous studies searching for the effect of anti-oxidant treatment on sperm DNA fragmentation incidence or DFI, up to our knowledge, this is the first study that evaluates the effects of anti-oxidant treatment on ICSI outcomes in normozoospermic patients with recurrent ICSI failure who have high DFI levels.

Data in this study suggest that anti-oxidant treatment for patients with high DFI levels, following unsuccessful ICSI attempts does not offer any advantage in terms of improving the ICSI outcomes. Yet it's difficult to conclude that anti-oxidant treatment has no beneficial effect in terms of decreasing ROS formation and improving fertility. The literature on the effect of anti-oxidant treatment on DNA fragmentation is conflicting. While some studies suggested beneficial effects, others reported a lack of improvement in the

Table 2. Cycle characteristics and cycle outcomes of study and control groups

\begin{tabular}{lccc}
\hline & $\begin{array}{c}\text { OA } \\
(\mathbf{n}=\mathbf{2 4})\end{array}$ & $\begin{array}{c}\text { ICSI-only } \\
(\mathbf{n}=\mathbf{4 6})\end{array}$ & $\boldsymbol{p}$ value \\
\hline Stimulation duration (days) & $11.26 \pm 1.25$ & $11.46 \pm 1.68$ & 0.610 \\
No of MII oocytes & $6.39 \pm 4.18$ & $7.24 \pm 2.45$ & 0.287 \\
Fertilization rate (\%) & $74.30 \pm 18.94$ & $70.30 \pm 20.26$ & 0.406 \\
Implantation rate (\%) & $26.50 \pm 6.61$ & $24.50 \pm 8.25$ & 0.176 \\
Clinical pregnancy, n (\%) & $9(37.5)$ & $9(19.6)$ & 0.103 \\
Abortion rate, $\mathrm{n}(\%)$ & $1(4.2)$ & $1(2.2)$ & 0.634 \\
\hline
\end{tabular}

Data are presented as mean \pm standard deviation. $\mathrm{OA}=$ oral antioxidant, ICSI $=$ intracytoplasmic sperm injection, $\mathrm{MII}=$ metaphase II 
outcomes. Menezo et al. [9] reported that anti-oxidant treatment led to a decrease in sperm fragmentation. An unexpected negative effect was also reported for antioxidant treatment which is an increase in sperm decondensation. The author concluded that this observation may be the cause of the discrepancy regarding the improvement in male fertility with antioxidant treatment [9]. In a similar way Amar et al. [10] stated that although anti-oxidant treatment limit ROS generation and related DNA damage, current antioxidant therapies might not be efficient and lead to decondensation and offered sequential treatment for handling the stiuation. On the contrary Greco et al. [11] suggested that antioxidant treatment reduces the proportion of spermatozoa with DNA damage and improves ICSI outcomes in men with high levels of sperm DNA fragmentation. However, similar to our study, no statistically significant difference was found in fertilization rates after anti-oxidant treatment. Of particular concern, in the study patients with no apperent improvement of ejaculated spermatazoa after the treatment were switched to an invasive alternative. Authors stated that; they did not address whether keeping up with the same treatment instead of switching to a more invasive one would make any difference and they also concluded this treatment may not be equally effective in a subgroup of patients who have no improvement after anti-oxidant treatment [11]. There are other studies consistent with the main finding of this study. Tunc et al. [12] reported that antioxidant treatment improves sperm DNA integrity. Greco et al. [13] reported that short term anti-oxidant supplementation is efficient for treating men with high sperm DNA damage. Abad et al. [14] suggested that in addition to improving semen quality in terms of key seminal parameters and basal DNA damage, antioxidant treatment also promotes better outcomes following assisted reproductive techniques. Gaul-Frau et al. [7] also showed beneficial effect of oral antioxidant treatment in grade 1 varicocele cases. However they also stated that despite better outcomes in terms of pregnancy by natural conception, higher rates of miscarriages were reported after oral antioxidant treatment [7].

\section{Limitations}

One of the limitations of our study apart from its retrospective nature is that the sample sizes were small. Literature in general keeps up with the hypothesis that anti-oxidant oral treatment appears to decrease the amount of sperm with DNA damage. However, mostly the effects are reported after short term treatment and there is no long-term follow-up results and the endpoint that is measured is sperm DNA integrity. Besides, most of the clinical studies have limited number of pariticipants and there is a great variation in the subpopulations. Therefore, this type of statement underlying the benefit derived from the anti-oxidant therapy needs to be balanced until more clinical studies with greater study populations are performed.

\section{CONCLUSION}

This study reveals that there is no statistically significant difference in the ICSI outcomes of the patients with high DFI levels, following unsuccessful ICSI attempts after oral anti-oxidant treatment.

\section{Author Contribution}

Research conception \& design: ST, GSÇ, AHH; Data acquisition/analysis and interpretation: EGP; MK; Statistical analysis: ST; Drafting of the manuscript: ST, GSÇ, MK, AHH; Critical revision of the manuscript and approval of final manuscript: ST, GSÇ, EGP, MK, AHH.

\section{Ethics statement}

Recently published The National Code on Clinical Trials has declared that ethics approval is not necessary for real retrospective studies. This is a retrospective study based on recorded data. Therefore approval from owners of the data (ST, GSÇ, EGP and $\mathrm{AHH}$ ) is obtained.

\section{Conflict of interest}

The authors disclosed no conflict of interest during the preparation or publication of this manuscript.

\section{Financing}

The authors disclosed that they did not receive any grant during conduction or writing of this study.

\section{Acknowledgements}

The authors thank IVF center nurses for technical 
assistance in data analysis.

\section{Disclosure}

Eczacıbaşı Pharmaceutical Company, Istanbul, Turkey has no influence on this work.

\section{REFERENCES}

[1] Zribi N, Chakroun NF, Elleuch H, Abdallah FB, Hamida ASB, Gargouri J, et al. Sperm DNA fragmentation and oxidation are independent of malondialdheyde. Reprod Biol Endocrinol 2011;9:47-55.

[2] Sakkas D, Alvarez JG. Sperm DNA fragmentation: mechanisms of origin, impact on reproductive outcome, and analysis. Fertil and Steril 2010;93:1027-36.

[3] Sharif K, Lewis EML. Debate Sperm DNA fragmentation testing: To do or not to do. Middle East Fertil Soc J 2013;18:7883.

[4] Robinson L, Gallos ID, Conner SJ, Rajkhowa M, Miller D, Lewis $\mathrm{S}$, et al. The effect of sperm DNA fragmentation on miscarriage rates: a systematic review and meta-analysis. Hum Reprod 2012;27:2908-17.

[5] More K, Badade ZG, Narshetty JG, Joshi DS, Mukherjee S, Deepak AD, et al. The effect of sperm DNA fragmentation on miscarriage rates: a systematic review and meta-analysis. J Mahatma Gandhi Inst Med Sci 2014;1:1-6.

[6] Lopes S, Jurisicova, Sun JG, Casper RF. Reactive oxygen species: potential cause for DNA fragmentation in human spermatozoa. Hum Reprod 1998;13:896-900.

[7] Gual-Frau J, Abad C, Amenguel MJ, Hannaoui N, Checa MA,
Ribas-Maynou J, et al. Oral antioxidant treatmen partly improves integrity of human sperm DNA in infertile grade 1 varicocele patients. Hum Fertil 2015;18:225-9.

[8] Martinez-Soto JC, Domingos JC, Cordobilla B, Nicolas M, Fernandez L, Albero P, et al. Dietary supplementation with docosahexaenoic acid (DHA) improves seminal antioxidant status and decreases sperm DNA fragmentation. Syst Biol Reprod Med 2016;62: 387-95.

[9] Menezo YR, Hazout A, Panteix G, Robert F, Rollet J, CohenBacrie P, et al. Antioxidants to reduce sperm DNA fragmentation: an unexpected adverse effect. Reprod Biomed Online 2007; 14:418-21.

[10] Amar E, Cornet D, Cohen M, Menezo Y. Treatment for hihg levels of sperm DNA fragmentation and nuclear decondensation: sequential treatment with a potent antioxidant followed by stimulation of the one-carbon cycle vs one-carbon cycle back-up alone. Austin J Reprod Med Infertil 2015;2:1-5.

[11] Greco E, Romano S, Iacobelli M, Ferrero S, Baroni E, Minasi MG, et al. ICSI in cases of sperm DNA damage: beneficial effect of oral antioxidant treatment. Hum Reprod 2005;20:2590-4.

[12] Tunc O, Thompson J, Tremellen K. Improvement in sperm DNA quality using an oral antioxidant therapy. Reprod Biomed Online 2009;18:761-8.

[13] Greco E, Iacobelli M, Rienzi L, Ubaldi F, Ferrero S, Tesarik J. Reduction of the incidence of sperm DNA fragmentation by oral antioxidant treatment. J Androl 2005;26:349-53.

[14] Abad C, Amengual MJ, Gosalvez J, Coward K, Hannaoui $\mathrm{N}$, Benet J, et al. Effects of oral antioxidant treatment upon the dynamics of human sperm DNA fragmentation and subpopulations of sperm with highly degraded DNA. Andrologia 2013;45:211-6. 